\title{
Cancer risks in a population-based study of Corsinati 70,570 agricultural workers: results from the Canadian census health and Environment cohort (CanCHEC)
}

\author{
Linda Kachuri ${ }^{1,2,3^{*}}$ (D, M. Anne Harris ${ }^{1,2,4}$, Jill S. MacLeod ${ }^{2,3}$, Michael Tjepkema ${ }^{5}$, Paul A. Peters ${ }^{6}$ and Paul A. Demers ${ }^{1,2,3}$
}

\begin{abstract}
Background: Agricultural workers may be exposed to potential carcinogens including pesticides, sensitizing agents and solar radiation. Previous studies indicate increased risks of hematopoietic cancers and decreased risks at other sites, possibly due to differences in lifestyle or risk behaviours. We present findings from CanCHEC (Canadian Census Health and Environment Cohort), the largest national population-based cohort of agricultural workers.

Methods: Statistics Canada created the cohort using deterministic and probabilistic linkage of the 1991 Canadian Long Form Census to National Cancer Registry records for 1992-2010. Self-reported occupations were coded using the Standard Occupational Classification (1991) system. Analyses were restricted to employed persons aged 25-74 years at baseline ( $N=2,051,315)$, with follow-up until December 31, 2010. Hazard ratios (HR) and 95\% confidence intervals (Cl) were modeled using Cox proportional hazards for all workers in agricultural occupations ( $n=70,570 ; 70.8 \%$ male), stratified by sex, and adjusted for age at cohort entry, province of residence, and highest level of education.
\end{abstract}

Results: A total of 9515 incident cancer cases (7295 in males) occurred in agricultural workers. Among men, increased risks were observed for non-Hodgkin lymphoma ( $\mathrm{HR}=1.10,95 \% \mathrm{Cl}=1.00-1.21)$, prostate $(\mathrm{HR}=1.11,95 \% \mathrm{Cl}=1.06-1$. 16), melanoma ( $H R=1.15,95 \% \mathrm{Cl}=1.02-1.31)$, and lip cancer ( $H R=2.14,95 \% \mathrm{Cl}=1.70-2.70)$. Decreased risks in males were observed for lung, larynx, and liver cancers. Among female agricultural workers there was an increased risk of pancreatic cancer ( $H R=1.36,95 \% \mathrm{Cl}=1.07-1.72)$. Increased risks of melanoma ( $H R=1.79,95 \% \mathrm{Cl}=1.17-2.73)$, leukemia $(\mathrm{HR}=2.01,95 \% \mathrm{Cl}=1.24-3.25)$ and multiple myeloma $(\mathrm{HR}=2.25,95 \% \mathrm{Cl}=1.16-4.37)$ were observed in a subset of female crop farmers.

Conclusions: Exposure to pesticides may have contributed to increased risks of hematopoietic cancers, while increased risks of lip cancer and melanoma may be attributed to sun exposure. The array of decreased risks suggests reduced smoking and alcohol consumption in this occupational group compared to the general population.

\section{Background}

Agricultural workers represent a unique population. While these individuals are employed in a range of occupations associated with exposure to a number of potential carcinogens, they also have a lower prevalence of cigarette smoking and increased levels of physical

\footnotetext{
* Correspondence: Linda.Kachuri@cancercare.on.ca

'Dalla Lana School of Public Health, University of Toronto, 155 College Street, 6th Floor, Toronto, ON M5T 3M7, Canada

${ }^{2}$ Occupational Cancer Research Centre, Cancer Care Ontario, 525 University

Avenue, 3rd Floor, Toronto, ON M5G 2L3, Canada

Full list of author information is available at the end of the article
}

activity [1]. As a result, individuals employed in agriculture tend to experience lower overall morbidity and mortality compared to the general population, and also exhibit distinctive cancer risk profiles [1-4]. Studies of cancer incidence and mortality in farmers have consistently reported reduced risks of tobacco-related cancers, such as lung and bladder [2, 3, 5-7]. Lower incidence of colorectal cancer has also been attributed to this favourable risk factor profile that includes higher levels of physical activity [3, 5, 7-9].

However, studies of agricultural workers also point to a number of elevated cancer risks in this population, 
especially for lymphatic and hematopoietic cancers, melanoma, lip cancer, prostate cancer and brain tumors $[2,3,5,7]$. This pattern is consistent with hazards associated with occupational exposure to pesticides, solvents, sunlight, dusts, and biological agents, such as zoonotic viruses, fungi and bacteria. Recent reports from the Agricultural Health Study (AHS), a large cohort of pesticide applicators and their spouses, also confirm many previous findings and implicate certain pesticides as key determinants of the cancer risks observed in agricultural populations [10].

Despite the accumulating evidence for a unique pattern of cancer incidence among agricultural workers and farmers, the last meta-analysis of studies in this area, conducted in 1998, reported inconsistent and highly heterogeneous results [3]. In addition to the multiplicity of exposures in agriculture, a possible explanation for these findings is that many earlier studies of agricultural populations have used mortality as the primary outcome, making it difficult to disentangle factors that influence cancer development from prognosis. Since then, few large-scale population-based occupational studies have been conducted, with one notable exception being the Nordic Occupational Cancer Study (NOCCA), which reported cancer incidence patterns among farmers and other occupational subgroups have been carried out in Denmark, Finland, Iceland, Norway and Sweden [5, 7]. However, similar comprehensive studies were lacking in Canada, despite the fact that agriculture has historically been one of the largest sectors of the Canadian economy.

A narrative review of the literature on cancer among farmers and an editorial dedicated to the NOCCA project, both published in 2009, called for future studies focusing on specific exposures, as well as the need for continued occupational cancer surveillance to document cancer incidence in agricultural workers and other occupational subgroups. [11, 12]. Furthermore, a consortium of agricultural cohort studies, AGRICOH, has been formed in 2010 to promote research efforts in this area, such as pooling of data across prospective studies to enable large-scale analyses of specific exposure-disease associations [13].

The Canadian Census Health and Environment Cohort (CanCHEC) was assembled to investigate cancer incidence patterns in specific socio-demographic and occupational groups in Canada. The aim of the present study was to address an important gap in Canadian occupational cancer surveillance by providing a comprehensive analysis of cancer risks among men and women employed in agriculture, by using data from a representative sample of the Canadian population. To our knowledge, this is the first and largest study of its kind in Canada, enabling a systematic analysis of cancer incidence by occupational subgroup, on a scale not previously possible in a single study.

\section{Methods \\ Study population}

The 1991-2010 Canadian Census Mortality and Cancer Follow-Up cohort is the foundational parent study for the Canadian Census Health and Environment Cohort (CanCHEC) [14, 15]. This cohort was originally created by linking the 1991 Canadian Census 2B (long form), with the Canadian Mortality Database (CMDB) and annual Historic Tax Summary Files (HTSF), to enable mortality follow-up from 1991 to 2001 [14, 15]. Individuals were eligible for the cohort if they were 25 years of age or older on Census day (June 4, 1991), were a usual resident of Canada, were not a long-term resident of an institution such as a prison, hospital or nursing home, and were among the $20 \%$ of Canadian households selected to complete the long-form census questionnaire.

The same inclusion criteria were maintained for CanCHEC, which was created by expanding the linkage of the 1991 Canadian Census to the Canadian Cancer Database (CCDB), in addition to updating the CMDB (1991-2011) and HTSF (1984-2011) data to 2011. Deterministic and probabilistic matching methods were used to link in-scope 1991 Census records to nonfinancial HTSF data, using dates of birth and postal codes of the individual and, if applicable, his or her spouse or common-law partner. The purpose of linkage to the HTSF files was to ascertain loss to follow-up, for instance due to a move outside of Canada. Individuals who stopped filing income taxes for 4 or more consecutive years by the end of the follow-up period, on December 31 2010, were identified using the HTSF and were considered lost to follow-up.

A total of 2.7 million individuals were successfully linked to the CMDB, equal to $15 \%$ of the Canadian noninstitutional resident population aged 25 years or older on Census day in 1991 [15]. Follow-up started on Census day in 1991 (June 4, 1991) and continued until December 31 2010. Demographic and socioeconomic information, and including age, sex, province or territory of residence, highest level of education, and occupation and industry were obtained from the 1991 long-form census.

\section{Ascertainment of cancer diagnosis}

Cancer diagnoses in CanCHEC were ascertained using data from the Canadian Cancer Database (CCDB). The CCDB combines two cancer data sources: the Canadian Cancer Registry (CCR) and the National Cancer Incidence Reporting System (NCIRS). The CCR is a personoriented tumor database that records the incidence of primary cancers diagnosed for each person since 1992 
[16]. Subsequent primary cancers diagnosed in patients who are already in the database are linked to their existing information. The NCIRS is a historical tumororiented database, which contains information on cancers diagnoses as far back as 1969 [16]. Individual cancer records from the CCR are used in the analysis, whereas historical information from NCIRS, linked to the CCR using probabilistic methods, was used to identify and exclude individuals with a cancer diagnosis within 10 years of cohort inception (1981-1991) in sensitivity analyses.

In our study, a case was defined as a primary incident cancer diagnosed between January 1, 1992 and December 31, 2010. Information on incident cancer diagnosis and year of diagnosis was retrieved from the CCR, and year of death was obtained from the CMDB, in order to remove deceased individuals from the cohort and ensure that the date of cancer diagnosis preceded the date of death. All cancers were defined using the 3rd revision of the International Classification of Diseases for Oncology (ICD-O-3) codes. With the exception of hematologic cancers (non-Hodgkin lymphoma, Hodgkin lymphoma, multiple myeloma, and leukemia) and mesothelioma, which were defined using morphology codes, the remaining cancer sites were identified using topography codes. Multiple cancers in the same individual were counted according to the International Agency for Research on Cancer (IARC) multiple primary rules [17].

This analysis examined a total of 25 diagnostic categories among male and female agricultural workers. For an assessment of overall cancer incidence, the "any cancer" category included all neoplasms, excluding nonmelanoma skin cancer. For individuals with multiple primary cancers, only the first diagnosis was counted in the any cancer category.

\section{Exposure assessment}

In the 1991 Census a respondent's occupation was determined by the job held in the week prior to the Census. If a participant reported no job in the last week, then the job with the longest duration since January 1, 1990 was recorded. If a participant had more than one job, then the job where most hours were worked was recorded as the main occupation.

Occupational data collected by Statistics Canada follows the structural framework of the Standard Occupational Classification (SOC). All occupations reported by the census participants were coded at Statistics Canada using the SOC 1991 system, and this forms the basis of the exposure assessment in our analysis. Within SOC91, occupations are grouped into 10 general categories, denoted by letters $\mathrm{A}-\mathrm{J}$, and occupational groups are further refined using 1-3 numbers. A total of 512 occupation groups are defined by the SOC 1991, which is based closely on its predecessor, the SOC 1980.

The specific SOC91 codes used to assess agricultural occupations and define exposure are presented in Table 1. In order to examine potential differences in risk between individuals employed in occupations that involve manual labour, the main exposure group was separated into two categories: farmers and managers (SOC91: I011-I016), and manual labourers (SOC91: I021, I022, I211, I212). In an effort to further refine the exposure groups by distinguishing between different types of agricultural work, classifications based on the SOC 80 coding system were used to identify crop farmers and workers (SOC80: 7115, 7185) and livestock farmers and workers (SOC80: 7113, 7183).

To minimize the healthy worker effect in CanCHEC, a working cohort was created by excluding individuals without a valid entry for occupation on the 1991 longform census [18]. This working cohort was further refined to minimize survival bias by excluding individuals over the age of 74 at baseline on June 4, 1991.

\section{Statistical analysis}

Hazard ratios (HRs) and corresponding 95\% confidence intervals (CI) were calculated using Cox proportional hazards regression to estimate cancer risks associated with employment in agriculture. Cox regression was selected over more crude approaches, such as standardization, because it allows for covariate adjustment, and unlike Poisson regression, the Cox model does not make parametric assumptions about the baseline hazard $[19,20]$.

The reference group consisted of all other employed cohort members, specifically, individuals not captured by the occupational groups in Table 1 . Models were adjusted for age at cohort entry (age group categories: 25-34, 35-44, 45-54, 55-74) and province of residence at the time of the Census. To control for potential confounding by socio-economic status, differences in screening rates, and lifestyle factors including cigarette smoking, physical activity, and diet, models were adjusted for highest attained level of education, which has been proposed as a suitable measure of socioeconomic status and proxy for life-style related risk factors $[21,22]$. We verified that none of the predictors violated the proportionality of hazards assumption. Stratified analyses were carried out to examine risks separately among men and women.

For each member of the cohort, follow-up time accrued from cohort entry on June 4, 1991 to date of cancer diagnosis, date of death, date of loss to follow-up or end of follow-up on December 31, 2010, whichever occurred first. Deceased individuals were identified in 
Table 1 Description of occupations used to define agricultural workers in CanCHEC (1991-2010)

\begin{tabular}{|c|c|c|c|c|c|c|}
\hline \multirow[t]{2}{*}{ Standard Occupational Classification (SOC) 1991 and 1980 Codes } & \multicolumn{2}{|l|}{ Males } & \multicolumn{2}{|c|}{ Females } & \multicolumn{2}{|l|}{ Total } \\
\hline & $\mathrm{N}$ & (\%) & $\mathrm{N}$ & (\%) & $\mathrm{N}$ & (\%) \\
\hline Farmers and managers (SOC91) & 33,980 & $(68.0)$ & 9385 & $(45.5)$ & 43,365 & (61.4) \\
\hline Farmers and farm managers (I011) & 30,215 & $(60.5)$ & 8415 & $(40.8)$ & 38,630 & $(54.7)$ \\
\hline Agricultural and related contractors and managers (1012) & 215 & $(0.4)$ & 20 & $(0.1)$ & 235 & (0.3) \\
\hline Farm supervisors and specialized livestock workers (1013) & 960 & $(1.9)$ & 315 & $(1.5)$ & 1275 & (1.8) \\
\hline Nursery and greenhouse operators and managers (1014) & 495 & $(1.0)$ & 355 & $(1.7)$ & 850 & $(1.2)$ \\
\hline Landscaping, ground maintenance contractors and managers (1015) & 925 & $(1.9)$ & 115 & (0.6) & 1040 & $(1.5)$ \\
\hline Landscape and horticulture supervisors (1016) & 1170 & $(2.3)$ & 165 & $(0.8)$ & 1335 & (1.9) \\
\hline Manual labourers (SOC91) & 15,985 & $(32.0)$ & 11,220 & $(54.5)$ & 27,205 & (38.6) \\
\hline General farm workers (1021) & 8945 & $(17.9)$ & 7620 & $(37.0)$ & 16,565 & $(23.5)$ \\
\hline Nursery and greenhouse workers (1022) & 1465 & $(2.9)$ & 1785 & $(8.7)$ & 3250 & $(4.6)$ \\
\hline Harvesting labourers (1211) & 485 & $(1.0)$ & 1020 & $(5.0)$ & 1505 & $(2.1)$ \\
\hline Landscaping and grounds maintenance labourers (1212) & 5090 & $(10.2)$ & 795 & $(3.9)$ & 5885 & $(8.4)$ \\
\hline \multicolumn{7}{|l|}{ Specialized subgroups (SOC80) } \\
\hline Crop farmers and farm workers $(7115,7185)$ & 2235 & $(4.5)$ & 3505 & $(17.0)$ & 5740 & $(8.1)$ \\
\hline Livestock farmers and farm workers $(7113,7183)$ & 2300 & $(4.6)$ & 1740 & $(8.4)$ & 4040 & (5.7) \\
\hline Total & 49,965 & (100.0) & 20,605 & $(100.0)$ & 70,570 & (100.0) \\
\hline
\end{tabular}

Note: counts below 5 have been suppressed and all counts have been randomly rounded to base 5 in accordance with Statistics Canada disclosure rules

the CMDB and removed from the at risk population in the cohort. Person-days were divided by 365.25 to obtain person-years at risk.

Data were accessed and analyzed in the secure facilities of the Toronto Research Data Centre located at Robarts Library, University of Toronto. Statistical analyses were performed using SAS 9.3/9.4 statistical software (SAS Institute Inc., Cary, NC, USA).

In accordance with Statistics Canada disclosure rules, case counts of less than 5 were suppressed in the reported tables, all frequencies were randomly rounded to the base 5 , and reported person-years at risk were rounded to the nearest 10 .

\section{Results}

\section{Population characteristics}

The derivation of the analytic cohort and flow of participants during the study period are illustrated in Fig. 1. Using the SOC91 occupation codes, we identified a total of 70,570 individuals (49,965 men, 20,605 women) employed in agriculture (Table 1). Farmers and managers constituted $61.4 \%$ of the main exposure group with 43,365 individuals, while the remaining 27,205 subjects (38.6\%) were classified as manual laborers. Examining this distribution by gender revealed that most male agricultural workers $(68.0 \%, 33,980$ subjects) were farmers and managers, whereas the majority of women $(54.5 \%, \quad 11,220$ subjects) worked in occupations associated with manual labour.
Using the SOC80 system, 5740 crop farmers and workers $(17 \%$ of female agricultural workers, $4.5 \%$ of male agricultural workers) and 4040 livestock farmers and workers (5.7\% of females, $4.6 \%$ of males) were also identified. Crop farmers and workers accounted for $47 \%$ of general farm workers (SOC91: I021), $20 \%$ of farmers and farm managers (SOC91: I011), 16\% of landscaping and grounds maintenance labourers (SOC91: I212), and 10\% of nursery and greenhouse workers (SOC91: I022). The majority of livestock farmers and workers (58\%) were included in the SOC91 general farm workers group (I021), followed by $28 \%$ in the farmers and farm managers category (SOC91: I011), and 6\% were part of the farm supervisors and specialized livestock workers (SOC91: I013) group. Overall, 92\% of crop farmers and workers and 93\% of livestock farmers and workers identified using SOC 80 were also captured by the SOC91 agricultural occupations.

Baseline demographic characteristics of CanCHEC are presented in Table 2. Compared to the entire working cohort, agricultural workers were older, predominantly male (70.8\%), and had a lower proportion of individuals with a university degree. Between cohort inception in 1991 and end of follow-up in December 2010, agricultural workers had accrued a total of 789,390 person-years of follow-up in males and 448,205 person-years in females. The mean personyears of follow-up were similar between agricultural workers (17.1 males, 17.9 females) and the entire 


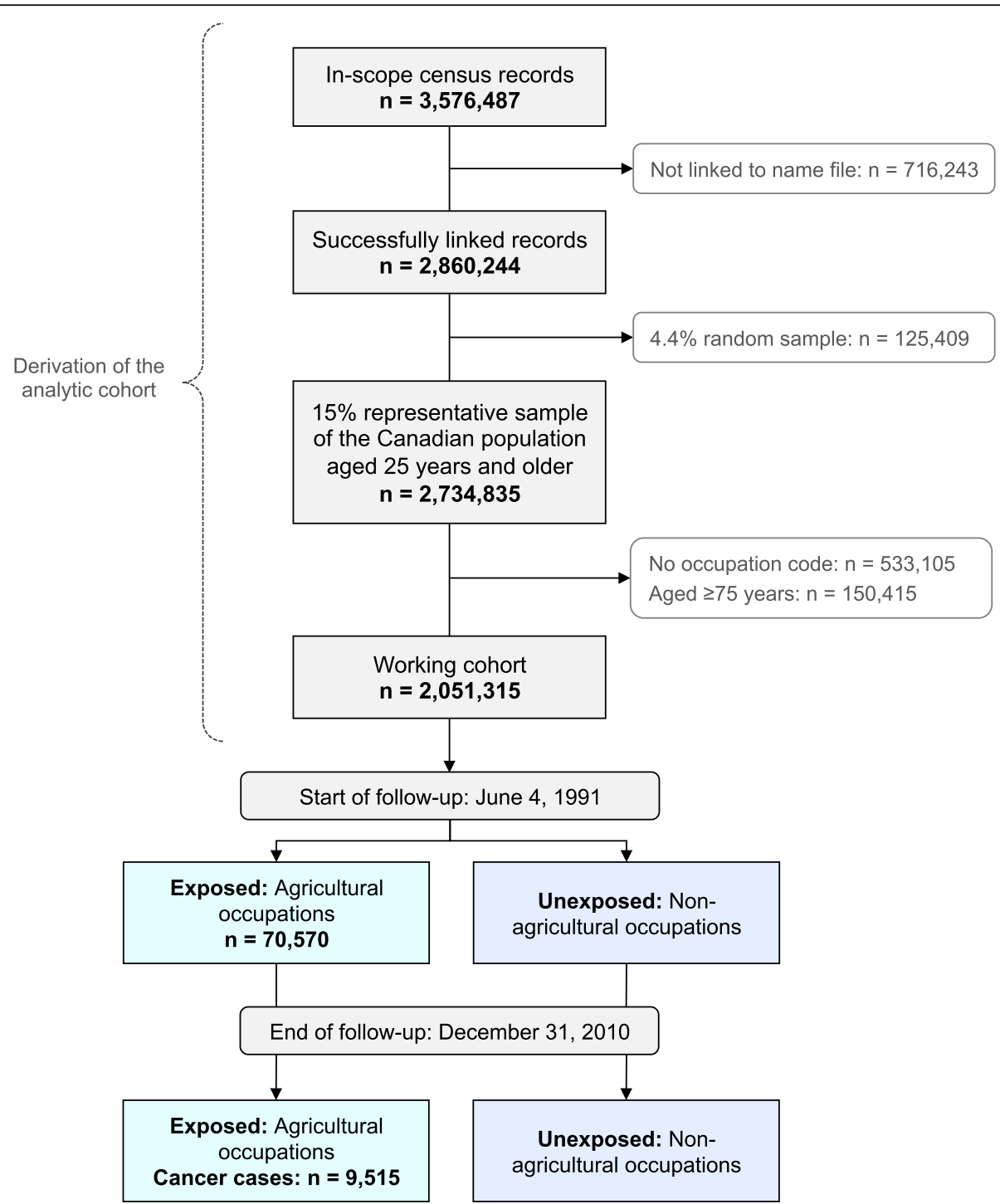

Fig. 1 Canadian Census Health and Environment Cohort (CanCHEC) participant flow and sample size based on the analytic cohort of derived from 1991 Census records

working CanCHEC sample (17.7 for males, 18.2 females).

Over the course of the follow-up period, a total of 9515 primary incident cancer cases were observed among agricultural workers. Of these, 7295 cases (76.7\%) were observed in men, who account for $70.8 \%$ of all agricultural workers, and 2220 cancers (23.3\%) occurred in women, who represent $29.2 \%$ of individuals employed in agriculture.

\section{Cancer risks in men}

Compared to the reference group, comprised of the working CanCHEC population, male agricultural workers were less likely to develop any type of malignancy $(\mathrm{HR}=0.95,95 \% \mathrm{CI}=0.93-0.98)$ (Table 3$)$. This inverse relationship remained statistically significant among farmers and managers $(\mathrm{HR}=0.94$, 95\% CI $=0.92-0.97)$, the larger occupational subgroup for male agricultural workers, and crop farmers $(\mathrm{HR}=0.84,95 \% \mathrm{CI}=0.75-0.95)$.

An array of decreased risks associated with employment in agriculture was observed across several sites, especially for cancers strongly linked to cigarette smoking and alcohol consumption (Table 3). Statistically significant inverse risk estimates were observed for cancer of the colon, liver, bladder, kidney, larynx, lung, and mesothelioma.

However, a number of statistically significant excesses in cancer risk were also observed among male agricultural workers in our cohort. The risk of being diagnosed with lip cancer was almost doubled for all agricultural workers $(\mathrm{HR}=2.14,95 \%$ 
Table 2 Baseline characteristics of CanCHEC (1991-2010) subjects aged 25 to 74 years at baseline, by employment status

\begin{tabular}{|c|c|c|c|c|c|c|c|c|}
\hline \multirow[t]{3}{*}{ Characteristics } & \multicolumn{4}{|c|}{ Agricultural Workers $(n=70,570)$} & \multicolumn{4}{|c|}{ Working Cohort $(n=2,051,315)$} \\
\hline & \multicolumn{2}{|l|}{ Males } & \multicolumn{2}{|l|}{ Females } & \multicolumn{2}{|l|}{ Males } & \multicolumn{2}{|l|}{ Females } \\
\hline & $\mathrm{N}$ & (\%) & $\mathrm{N}$ & (\%) & $\mathrm{N}$ & (\%) & $\bar{N}$ & (\%) \\
\hline \multicolumn{9}{|l|}{ Age at entry to cohort } \\
\hline Mean age in years (SD) & 46.4 & $(13.7)$ & 44.8 & $(12.5)$ & 41.7 & $(11.3)$ & 40.2 & $(10.5)$ \\
\hline $25-34$ years & 12,840 & $(25.7)$ & 5355 & $(26.0)$ & 359,075 & $(32.4)$ & 336,870 & $(35.7)$ \\
\hline $35-44$ years & 11,640 & $(23.3)$ & 5500 & $(26.7)$ & 341,515 & $(30.8)$ & 309,600 & $(32.8)$ \\
\hline $45-54$ years & 9245 & $(18.5)$ & 4640 & $(22.5)$ & 229,460 & $(20.7)$ & 187,465 & $(19.9)$ \\
\hline $55-65$ years & 10,195 & $(20.4)$ & 3520 & $(17.1)$ & 143,895 & $(13.0)$ & 91,135 & $(9.7)$ \\
\hline $65-74$ years & 6045 & $(12.1)$ & 1590 & $(7.7)$ & 34,465 & (3.1) & 17,845 & (1.9) \\
\hline \multicolumn{9}{|l|}{ Province of residence } \\
\hline Newfoundland and Labrador & 250 & $(0.5)$ & 80 & $(0.4)$ & 21,815 & $(2.0)$ & 17,530 & (1.9) \\
\hline Prince Edward Island & 500 & $(1.0)$ & 165 & $(0.8)$ & 4945 & $(0.4)$ & 4475 & $(0.5)$ \\
\hline Nova Scotia & 900 & (1.8) & 370 & $(1.8)$ & 34,750 & (3.1) & 28,405 & (3.0) \\
\hline New Brunswick & 800 & (1.6) & 350 & $(1.7)$ & 27,600 & $(2.5)$ & 22,615 & $(2.4)$ \\
\hline Quebec & 7845 & $(15.7)$ & 3070 & $(14.9)$ & 276,120 & $(24.9)$ & 226,375 & $(24.0)$ \\
\hline Ontario & 12,290 & $(24.6)$ & 5375 & $(26.1)$ & 404,130 & $(36.5)$ & 352,165 & $(37.3)$ \\
\hline Manitoba & 4545 & (9.1) & 1525 & $(7.4)$ & 47,375 & $(4.3)$ & 40,190 & $(4.3)$ \\
\hline Saskatchewan & 9395 & $(18.8)$ & 3320 & $(16.1)$ & 42,050 & (3.8) & 35,805 & (3.8) \\
\hline Alberta & 9295 & $(18.6)$ & 3875 & $(18.8)$ & 107,405 & $(9.7)$ & 92,730 & $(9.8)$ \\
\hline British Columbia & 4045 & $(8.1)$ & 2455 & $(11.9)$ & 130,815 & $(11.8)$ & 112,920 & $(12.0)$ \\
\hline Yukon, Northwest Territories, Nunavut & 100 & $(0.2)$ & 20 & $(0.1)$ & 11,395 & $(1.0)$ & 9705 & $(1.0)$ \\
\hline \multicolumn{9}{|l|}{ Highest level of education } \\
\hline No high school diploma & 27,080 & $(54.2)$ & 9785 & $(47.5)$ & 322,190 & $(29.1)$ & 233,565 & $(24.8)$ \\
\hline High school or trade certificate & 16,440 & $(32.9)$ & 7150 & $(34.7)$ & 444,560 & $(40.1)$ & 360,215 & $(38.2)$ \\
\hline Post-secondary non-university & 4345 & $(8.7)$ & 2845 & $(13.8)$ & 154,165 & $(13.9)$ & 207,865 & $(22.0)$ \\
\hline University degree & 2100 & $(4.2)$ & 825 & $(4.0)$ & 187,495 & $(16.9)$ & 141,260 & (15.0) \\
\hline Subtotal & 49,965 & $(70.8)^{a}$ & 20,605 & $(29.2)^{a}$ & $1,108,410$ & $(54.0)^{b}$ & 942,905 & $(46.0)^{b}$ \\
\hline $\begin{array}{l}\text { Person-years of follow-up } \\
\text { Total (Mean) }\end{array}$ & 789,390 & $(17.1)$ & 448,205 & $(17.9)$ & $19,635,045$ & $(17.7)$ & $17,116,840$ & (18.2) \\
\hline
\end{tabular}

Note: case counts below 5 have been suppressed and all counts have been randomly rounded to base 5 in accordance with Statistics Canada disclosure rules a Percentage calculated from total number of agricultural workers $(n=70,570)$

${ }^{b}$ Percentage calculated from total number of subjects in working cohort $(n=2,051,315)$

$\mathrm{CI}=1.70-2.70)$, farmers and managers $(\mathrm{HR}=2.25$, 95\% $\mathrm{CI}=1.75-2.90)$, and manual labourers $(\mathrm{HR}=1.80,95 \% \mathrm{CI}=1.15-2.84)$. A statistically significant increase in melanoma risk was also observed among all males in agriculture $(\mathrm{HR}=1.15,95 \%$ $\mathrm{CI}=1.02-1.31)$, and farmers and managers $(\mathrm{HR}=1.21,95 \% 1.05-1.39)$.

Other notable increases in risk were observed for prostate cancer and non-Hodgkin lymphoma (NHL). The increase in the risk of prostate cancer was observed for all agricultural workers $(\mathrm{HR}=1.11,95 \% \mathrm{CI}=1.06-1.16)$, farmers and managers $(\mathrm{HR}=1.12,95 \% \mathrm{CI}=1.07-1.17)$, and manual labourers $(\mathrm{HR}=1.08,95 \% \mathrm{CI}=0.99-1.17)$. The increase in NHL risk was similar in magnitude, but reached marginal significance only in the overall exposure group $(\mathrm{HR}=1.10,95 \% \mathrm{CI}=1.00-1.21)$.

Investigating more refined agricultural subgroups revealed several notable increases in risk among crop farmers (Table 5) and livestock farmers (Table 6). Mirroring the main analyses, risks of lip cancer remained elevated for both crop (HR $=3.59,95 \% \mathrm{CI}=1.69-7.62)$ and livestock farmers $(\mathrm{HR}=2.91,95 \% \mathrm{CI}=1.08-7.80)$. The risk of prostate cancer was elevated only in the livestock subgroup $(\mathrm{HR}=1.26,95 \% \mathrm{CI}=1.03-1.55)$. Despite a small number of cases, a large and statistically significant increase in thyroid cancer risk $(\mathrm{HR}=3.01$, $95 \% \mathrm{CI}=1.35-6.73)$ was also observed among livestock farmers. 
Table 3 Hazard ratios (HR) and 95\% confidence intervals (Cl) for selected cancers among male agricultural workers in CanCHEC (1991-2010)

\begin{tabular}{|c|c|c|c|c|c|c|c|c|c|}
\hline \multirow[t]{2}{*}{ Cancer Site (ICD-O-3) } & \multicolumn{3}{|c|}{ Agricultural Workers } & \multicolumn{3}{|c|}{ Farmers and managers } & \multicolumn{3}{|c|}{ Manual labourers } \\
\hline & Cases & $\mathrm{HR}$ & $(95 \% C l)^{a}$ & Cases & $\mathrm{HR}$ & $(95 \% C l)^{a}$ & Cases & $\mathrm{HR}$ & $(95 \% C l)^{a}$ \\
\hline Any cancer ${ }^{\mathrm{b}}$ & 7295 & 0.95 & $(0.93-0.98)$ & 5415 & 0.94 & $(0.92-0.97)$ & 1880 & 0.98 & $(0.93-1.02)$ \\
\hline Prostate (C61.9) & 2625 & 1.11 & $(1.06-1.16)$ & 2025 & 1.12 & $(1.07-1.17)$ & 600 & 1.08 & $(0.99-1.17)$ \\
\hline Lung (C34) & 995 & 0.75 & $(0.70-0.80)$ & 690 & 0.69 & $(0.64-0.75)$ & 305 & 0.90 & $(0.81-1.01)$ \\
\hline Colon (C18, C26.0) & 640 & 0.89 & $(0.82-0.97)$ & 485 & 0.90 & $(0.82-0.99)$ & 155 & 0.88 & $(0.75-1.03)$ \\
\hline Rectum (C19.9, C20.9) & 440 & 1.05 & $(0.95-1.16)$ & 340 & 1.09 & $(0.97-1.22)$ & 100 & 0.94 & $(0.77-1.15)$ \\
\hline Non-Hodgkin Lymphoma ${ }^{c}$ & 500 & 1.10 & $(1.00-1.21)$ & 375 & 1.11 & $(0.99-1.24)$ & 125 & 1.09 & $(0.91-1.30)$ \\
\hline Bladder (C67) & 430 & 0.82 & $(0.74-0.91)$ & 325 & 0.83 & $(0.73-0.93)$ & 105 & 0.82 & $(0.67-1.00)$ \\
\hline Melanoma (C44) & 275 & 1.15 & $(1.02-1.31)$ & 215 & 1.21 & $(1.05-1.39)$ & 60 & 1.00 & $(0.77-1.29)$ \\
\hline Leukemia $^{c}$ & 265 & 1.11 & $(0.97-1.27)$ & 200 & 1.14 & $(0.98-1.32)$ & 65 & 1.03 & $(0.80-1.32)$ \\
\hline Oral (COO-C14) & 250 & 1.04 & $(0.91-1.19)$ & 170 & 0.97 & $(0.82-1.14)$ & 80 & 1.24 & $(0.99-1.55)$ \\
\hline Lip (C00.0-C00.9) & 110 & 2.14 & $(1.70-2.70)$ & 90 & 2.25 & $(1.75-2.90)$ & 20 & 1.80 & $(1.15-2.84)$ \\
\hline Kidney (C64.9) & 230 & 0.77 & $(0.67-0.89)$ & 175 & 0.78 & $(0.67-0.92)$ & 55 & 0.74 & $(0.57-0.97)$ \\
\hline Stomach (C16) & 225 & 0.90 & $(0.78-1.04)$ & 160 & 0.87 & $(0.74-1.03)$ & 65 & 0.99 & $(0.77-1.26)$ \\
\hline Pancreas (C25) & 175 & 0.90 & $(0.77-1.06)$ & 125 & 0.86 & $(0.71-1.03)$ & 50 & 1.03 & $(0.77-1.36)$ \\
\hline Multiple myelomac & 135 & 1.15 & $(0.95-1.38)$ & 100 & 1.14 & $(0.92-1.41)$ & 35 & 1.17 & $(0.84-1.64)$ \\
\hline Brain (C70-C72) & 110 & 0.89 & $(0.73-1.08)$ & 75 & 0.84 & $(0.66-1.06)$ & 35 & 1.01 & $(0.72-1.41)$ \\
\hline Esophagus (C15) & 95 & 0.87 & $(0.70-1.08)$ & 65 & 0.78 & $(0.60-1.00)$ & 30 & 1.15 & $(0.80-1.65)$ \\
\hline Thyroid (C73.9) & 60 & 1.05 & $(0.79-1.38)$ & 45 & 1.06 & $(0.77-1.47)$ & 15 & 1.00 & $(0.59-1.70)$ \\
\hline Larynx (C32) & 55 & 0.54 & $0.41-0.71)$ & 30 & 0.39 & $(0.27-0.56)$ & 25 & 0.96 & $(0.64-1.43)$ \\
\hline Liver (C22.0, C22.1) & 45 & 0.51 & $(0.38-0.68)$ & 30 & 0.43 & $(0.30-0.62)$ & 15 & 0.75 & $(0.47-1.20)$ \\
\hline Testis (C62) & 30 & 0.97 & $(0.69-1.37)$ & 20 & 1.02 & $(0.66-1.56)$ & 10 & 0.90 & $(0.52-1.56)$ \\
\hline Hodgkin Lymphoma $^{c}$ & 25 & 0.78 & $(0.49-1.25)$ & 15 & 0.66 & $(0.36-1.22)$ & 10 & 1.02 & $(0.51-2.07)$ \\
\hline Mesothelioma $^{c}$ & 20 & 0.57 & $(0.36-0.90)$ & 15 & 0.56 & $(0.33-0.95)$ & 5 & 0.59 & $(0.24-1.43)$ \\
\hline Breast (C50) & 20 & 1.27 & $(0.74-2.15)$ & 10 & 0.96 & $(0.48-1.91)$ & 10 & 2.08 & $(0.97-4.44)$ \\
\hline Nasal (C30) & 15 & 0.71 & $(0.39-1.29)$ & 10 & 0.77 & $(0.38-1.53)$ & 5 & 0.60 & $(0.22-1.65)$ \\
\hline Bone (C40, C41) & 5 & 0.69 & $(0.36-1.32)$ & $<5$ & - & - & $<5$ & - & - \\
\hline
\end{tabular}

Note: case counts below 5 have been suppressed and all counts have been randomly rounded to base 5 in accordance with Statistics Canada disclosure rules ${ }^{a}$ Adjusted for age at baseline (age group categories), province of residence at baseline, and education level at baseline

b Incident primary cancers excluding non-melanoma skin cancer

'Cancers defined using ICD-O-3 Histology codes: Mesothelioma (9050-9055), Hodgkin lymphoma (9650-9667); non-Hodgkin lymphoma (9590-9596,9670-9719, 9727-9729, 9823, 9827); Multiple myeloma (9731,9732,9734); Leukemia (9733, 9742, 9800-9801, 9805, 9820, 9826, 9831-9837, 9840, 9860-9861, 9863, 9866-9867, 9870-9876,9891,9895-9897,9910, 9920, 9930-9931, 9940, 9945-9946, 9948, 9963-9964, 9823, 9827)

\section{Cancer risks in women}

Similar to the pattern observed for men, women employed in agriculture appeared to have a decreased overall cancer risk $(\mathrm{HR}=0.9295 \% \mathrm{CI}=0.88-0.96)$. The inverse association between agricultural occupation and cancer risk remained statistically significant in the larger manual laborers subgroup $(\mathrm{HR}=0.88$, 95\% CI $=0.83-0.93)$, and among crop farmers $(\mathrm{HR}=0.85,95 \% \mathrm{CI}=0.76-0.94)$.

In contrast to the multiple negative associations observed in men, only lung cancer exhibited inverse risk estimates among women across most exposure groups (Table 4). This negative association was statistically significant for all agricultural occupations ( $\mathrm{HR}=0.58,95 \%$ $\mathrm{CI}=0.50-0.66)$, farmers and managers $(\mathrm{HR}=0.57,95 \%$
$\mathrm{CI}=0.47-0.70)$, and manual labourers $(\mathrm{HR}=0.58,95 \%$ $\mathrm{CI}=0.48-0.70)$. In addition, belonging to the manual labourer subgroup was also associated with a decreased risk of colon cancer $(\mathrm{HR}=0.73,95 \% \mathrm{CI}=0.58-0.91)$. Notably, the pattern of colon cancer risk among farmers and managers was in the opposite direction $(\mathrm{HR}=1.19$, 95\% CI $=0.99-1.42$ ) in women compared to men.

Few statistically significant increases in risk were observed among female agricultural workers (Table 4). The incidence of pancreatic cancer was significantly higher among all females employed in agriculture $(\mathrm{HR}=1.36$, $95 \% \mathrm{CI}=1.07-1.72)$, and manual labourers $(\mathrm{HR}=1.45$, $95 \%$ CI $=1.05-2.01)$. Risk of leukemia was also significantly elevated among farmers and managers $(\mathrm{HR}=1.70$, 95\% CI $=1.26-2.29$ ). 
Table 4 Hazard ratios (HR) and 95\% confidence intervals (Cl) for selected cancers among female agricultural workers in CanCHEC (1991-2010)

\begin{tabular}{|c|c|c|c|c|c|c|c|c|c|}
\hline \multirow[t]{2}{*}{ Cancer Site (ICD-O-3) } & \multicolumn{3}{|c|}{ Agricultural Workers } & \multicolumn{3}{|c|}{ Farmers and managers } & \multicolumn{3}{|c|}{ Manual labourers } \\
\hline & Cases & $\mathrm{HR}$ & $(95 \% \mathrm{Cl})^{\mathrm{a}}$ & Cases & $\mathrm{HR}$ & $(95 \% C l)^{a}$ & Cases & $\mathrm{HR}$ & $(95 \% C l)^{\mathrm{a}}$ \\
\hline Any cancer ${ }^{b}$ & 2220 & 0.92 & $(0.88-0.96)$ & 1130 & 0.96 & $(0.90-1.02)$ & 1090 & 0.88 & $(0.83-0.93)$ \\
\hline Breast (C50) & 700 & 0.92 & $(0.85-0.99)$ & 350 & 0.92 & $(0.83-1.03)$ & 350 & 0.92 & $(0.83-1.02)$ \\
\hline Lung (C34) & 210 & 0.58 & $(0.50-0.66)$ & 100 & 0.57 & $(0.47-0.70)$ & 110 & 0.58 & $(0.48-0.70)$ \\
\hline Colon (C18, C26.0) & 205 & 0.95 & $(0.82-1.10)$ & 120 & 1.19 & $(0.99-1.42)$ & 80 & 0.73 & $(0.58-0.91)$ \\
\hline Rectum (C19.9, C20.9) & 100 & 1.08 & $(0.88-1.32)$ & 45 & 1.08 & $(0.81-1.45)$ & 55 & 1.07 & $(0.81-1.42)$ \\
\hline Non-Hodgkin Lymphomac & 135 & 1.02 & $(0.86-1.22)$ & 75 & 1.17 & $(0.92-1.47)$ & 65 & 0.89 & $(0.69-1.15)$ \\
\hline Melanoma (C44) & 90 & 1.14 & $(0.93-1.41)$ & 40 & 1.00 & $(0.72-1.39)$ & 55 & 1.26 & $(0.96-1.66)$ \\
\hline Ovary (C56.9) & 80 & 0.99 & $(0.79-1.23)$ & 75 & 0.90 & $(0.65-1.25)$ & 45 & 1.07 & $(0.79-1.43)$ \\
\hline Leukemia $^{c}$ & 75 & 1.21 & $(0.95-1.54)$ & 45 & 1.70 & $(1.26-2.29)$ & 30 & 0.82 & $(0.56-1.20)$ \\
\hline Thyroid (C73.9) & 75 & 1.25 & $(0.99-1.59)$ & 35 & 1.25 & $(0.88-1.77)$ & 40 & 1.26 & $(0.92-1.72)$ \\
\hline Pancreas (C25) & 75 & 1.36 & $(1.07-1.72)$ & 40 & 1.27 & $(0.91-1.77)$ & 35 & 1.45 & $(1.05-2.01)$ \\
\hline Bladder (C67) & 50 & 0.88 & $(0.65-1.17)$ & 20 & 0.86 & $(0.56-1.32)$ & 30 & 0.89 & $(0.60-1.31)$ \\
\hline Kidney (C64.9) & 45 & 0.79 & $(0.58-1.08)$ & 25 & 0.88 & $(0.59-1.32)$ & 15 & 0.70 & $(0.44-1.12)$ \\
\hline Brain (C70-C72) & 45 & 1.26 & $(0.92-1.71)$ & 20 & 1.42 & $(0.94-2.16)$ & 20 & 1.11 & $(0.71-1.73)$ \\
\hline Cervix (C53) & 40 & 0.83 & $(0.60-1.15)$ & 10 & 0.69 & $(0.41-1.18)$ & 25 & 0.94 & $(0.63-1.41)$ \\
\hline Stomach (C16) & 35 & 0.92 & $(0.65-1.31)$ & 20 & 1.14 & $(0.73-1.79)$ & 10 & 0.71 & $(0.41-1.22)$ \\
\hline Oral (CO0-C14) & 35 & 0.79 & $(0.55-1.13)$ & 15 & 0.80 & $(0.50-1.29)$ & 15 & 0.78 & $(0.46-1.32)$ \\
\hline Lip (C00.0-C00.9) & $<5$ & - & - & $<5$ & - & - & $<5$ & - & - \\
\hline Multiple Myelomac & 25 & 0.81 & $(0.54-1.21)$ & 10 & 0.54 & $(0.30-0.99)$ & 15 & 1.24 & $(0.74-2.08)$ \\
\hline Liver (C22.0, C22.1) & 15 & 0.90 & $(0.51-1.57)$ & 5 & 0.88 & $(0.41-1.88)$ & 5 & 0.92 & $(0.41-2.06)$ \\
\hline Esophagus (C15) & 10 & 1.06 & $(0.60-1.87)$ & 10 & 1.40 & $(0.65-2.98)$ & 5 & 0.82 & $(0.36-1.88)$ \\
\hline Mesotheliomac & 5 & 1.14 & $(0.37-3.47)$ & $<5$ & - & - & $<5$ & - & - \\
\hline Nasal (C30) & $<5$ & - & - & $<5$ & - & - & $<5$ & - & - \\
\hline Larynx (C32) & $<5$ & - & - & $<5$ & - & - & $<5$ & - & - \\
\hline Bone (C40, C41) & $<5$ & - & - & $<5$ & - & - & $<5$ & - & - \\
\hline Hodgkin Lymphomac & $<5$ & - & - & $<5$ & - & - & $<5$ & - & - \\
\hline
\end{tabular}

Note: case counts below 5 have been suppressed and all counts have been randomly rounded to base 5 in accordance with Statistics Canada disclosure rules ${ }^{a}$ Adjusted for age at baseline (age group categories), province of residence at baseline, and education level at baseline

b Incident primary cancers excluding non-melanoma skin cancer

'Cancers defined using ICD-O-3 Histology codes: Mesothelioma (9050-9055), Hodgkin lymphoma (9650-9667); non-Hodgkin lymphoma (9590-9596,9670-9719, 9727-9729, 9823, 9827); Multiple myeloma (9731,9732,9734); Leukemia (9733, 9742, 9800-9801, 9805, 9820, 9826, 9831-9837, 9840, 9860-9861, 9863, 9866-9867, 9870-9876,9891,9895-9897,9910, 9920, 9930-9931, 9940, 9945-9946, 9948, 9963-9964, 9823, 9827)

Elevated risks that did not reach statistical significance were observed for thyroid cancer in all female agricultural workers $(\mathrm{HR}=1.25,95 \% \mathrm{CI}=0.99-1.59)$, melanoma among manual labourers $(\mathrm{HR}=1.26,95 \% \mathrm{CI}=0.96-$ $1.66)$, and brain tumors in the farmer and manager subgroup $(\mathrm{HR}=1.42,95 \% \mathrm{CI}=0.94-2.16)$.

Several associations emerged from more refined analyses of crop farmers (Table 5) and livestock farmers (Table 6). Crop farmers had an approximately 2 -fold increase in the risk of multiple myeloma $(\mathrm{HR}=2.25,95 \% \mathrm{CI}=1.16$ 4.37), leukemia ( $\mathrm{HR}=2.01,95 \% \mathrm{CI}=1.24-3.25)$, and melanoma $(\mathrm{HR}=1.79,95 \% \mathrm{CI}=1.17-2.73)$. Although based on a small number of cases, significant excesses in oral cancer risk were observed among women working as livestock farmers $(\mathrm{HR}=2.70,95 \% \mathrm{CI}=1.28-5.68)$.

\section{Sensitivity analyses}

In an effort to refine the pattern of cancer risks observed for agricultural workers, we carried out several stratified analyses. First, a sub-cohort was created by excluding individuals with a cancer diagnosis within 10 years of cohort inception, between 1981 and 1991. These exclusions removed approximately 28,680 individuals from the entire working cohort, including prevalent cases and individuals with a past history of cancer. Associations with agricultural occupations were estimated separately for men (Additional file 1: Table S1) and women (Additional file 2: Table S2).

The pattern and magnitude of cancer risks among agricultural workers in the cancer-free sub-cohort closely resembled those observed in the main analysis. 
Table 5 Hazard ratios (HR) and 95\% confidence intervals (Cl) for selected cancers among male and female crop farmers and farm workers in CanCHEC (1991-2010)

\begin{tabular}{|c|c|c|c|c|c|c|}
\hline \multirow[t]{2}{*}{ Cancer Site (ICD-O-3) } & \multicolumn{3}{|l|}{ Men } & \multicolumn{3}{|c|}{ Women } \\
\hline & Cases & $\mathrm{HR}$ & $(95 \% \mathrm{Cl})^{\mathrm{a}}$ & Cases & $\mathrm{HR}$ & $(95 \% \mathrm{Cl})^{\mathrm{a}}$ \\
\hline Any cancer ${ }^{b}$ & 270 & 0.84 & $(0.75-0.95)$ & 355 & 0.85 & $(0.76-0.94)$ \\
\hline Breast (C50) & 0 & - & - & 110 & 0.85 & $(0.70-1.02)$ \\
\hline Prostate (C61.9) & 80 & 0.82 & $(0.66-1.02)$ & - & - & - \\
\hline Leukemia3 & 80 & 1.08 & $(0.60-1.95)$ & 15 & 2.01 & $(1.24-3.25)$ \\
\hline Lung (C34) & 50 & 0.85 & $(0.64-1.13)$ & 30 & 0.44 & $(0.31-0.63)$ \\
\hline Melanoma (C44) & $<5$ & - & - & 25 & 1.79 & $(1.17-2.73)$ \\
\hline Colon (C18, C26.0) & 30 & 1.10 & $(0.77-1.56)$ & 20 & 0.62 & $(0.39-0.97)$ \\
\hline Rectum (C19.9, C20.9) & 15 & 0.88 & $(0.53-1.46)$ & 15 & 0.96 & $(0.58-1.59)$ \\
\hline Non-Hodgkin Lymphomac & 15 & 1.16 & $(0.48-2.80)$ & 15 & 0.74 & $(0.47-1.18)$ \\
\hline Bladder (C67) & 15 & 0.75 & $(0.47-1.20)$ & $<5$ & - & - \\
\hline Thyroid (C73.9) & 0 & - & - & 15 & 1.55 & $(0.96-2.51)$ \\
\hline Ovary (C56.9) & - & - & - & 15 & 1.08 & $(0.66-1.77)$ \\
\hline Cervix (C53) & - & - & - & 10 & 1.13 & $(0.59-2.18)$ \\
\hline Multiple Myeloma ${ }^{c}$ & 10 & 0.84 & $(0.52-1.38)$ & 10 & 2.25 & $(1.16-4.37)$ \\
\hline Kidney (C64.9) & 10 & 0.90 & $(0.48-1.68)$ & 10 & 0.82 & $(0.39-1.74)$ \\
\hline Stomach (C16) & 10 & 0.61 & $(0.32-1.18)$ & 10 & 1.05 & $(0.50-2.21)$ \\
\hline Oral (COO-C14) & 10 & 1.26 & $(0.71-2.22)$ & 5 & 0.82 & $(0.34-1.98)$ \\
\hline Lip (C00.0-C00.9) & 10 & 3.59 & $(1.69-7.62)$ & 0 & - & - \\
\hline Pancreas (C25) & 5 & 0.87 & $(0.41-1.83)$ & 5 & 1.02 & $(0.53-1.96)$ \\
\hline Brain (C70-C72) & $<5$ & - & - & 5 & 1.06 & $(0.47-2.37)$ \\
\hline Larynx (C32) & 5 & 1.50 & $(0.67-3.36)$ & 0 & - & - \\
\hline Bone (C40, C41) & $<5$ & - & - & $<5$ & - & - \\
\hline Liver (C22.0, C22.1) & $<5$ & - & - & 0 & - & - \\
\hline Nasal (C30) & $<5$ & - & - & 0 & - & - \\
\hline Esophagus (C15) & 0 & - & - & $<5$ & - & - \\
\hline Mesothelioma ${ }^{c}$ & 0 & - & - & $<5$ & - & - \\
\hline Hodgkin Lymphoma & 0 & - & - & $<5$ & - & - \\
\hline Testis (C62) & 0 & - & - & - & - & - \\
\hline
\end{tabular}

Note: case counts below 5 have been suppressed and all counts have been randomly rounded to base 5 in accordance with Statistics Canada disclosure rules ${ }^{a}$ Adjusted for age at baseline (age group categories), province of residence at baseline, and education level at baseline

${ }^{b}$ Incident primary cancers excluding non-melanoma skin cancer

'Cancers defined using ICD-O-3 Histology codes: Mesothelioma (9050-9055), Hodgkin lymphoma (9650-9667); non-Hodgkin lymphoma (9590-9596,9670-9719, 9727-9729, 9823, 9827); Multiple myeloma (9731,9732,9734); Leukemia (9733, 9742, 9800-9801, 9805, 9820, 9826, 9831-9837, 9840, 9860-9861, 9863, 9866-9867, 9870-9876,9891,9895-9897,9910, 9920, 9930-9931, 9940, 9945-9946, 9948, 9963-9964, 9823, 9827)

Risk of oral cavity cancers was only elevated in the labourer group ( $\mathrm{HR}=1.25$, 95\% CI: 1.00-1.57). However, an approximately 2-fold increase in lip cancer risk was observed for all male agricultural workers $(\mathrm{HR}=2.10$, 95\% CI: $1.65-2.66)$, farmers and managers $(\mathrm{HR}=2.17$, 95\% CI: $1.67-2.82$ ) and manual labourers ( $\mathrm{HR}=1.85$, 95\% CI: $1.18-2.92)$. The previously observed $11 \%$ and $12 \%$ increase in prostate cancer risk overall, and among farmers and managers, persisted in the cancer-free subcohort. A similar pattern was observed for melanoma, with $15 \%$ greater risks observed for agricultural work overall and a $21 \%$ increase in risk observed in farmers and managers. Although based on a small number of cases, the risk of male breast cancer was more than double among workers and labourers in agriculture $(\mathrm{HR}=2.14,95 \% \mathrm{CI}: 1.00-4.58)$.

The exclusion of pre-1991 cancer cases did not alter the pattern of associations observed for women employed in agriculture (Additional file 2: Table S2). The increase in leukemia risk was the highest among farmers and managers $(\mathrm{HR}=1.74,95 \% \mathrm{CI}: 1.29-2.35)$, and somewhat attenuated in the overall exposure group $(\mathrm{HR}=1.24,95 \% \mathrm{CI}:$ 0.98-1.58). Risk of pancreatic cancer was $42 \%$ greater among female labourers and $32 \%$ 
Table 6 Hazard ratios (HR) and 95\% confidence intervals (Cl) for selected cancers among male and female livestock farmers and farm workers in CanCHEC (1991-2010)

\begin{tabular}{|c|c|c|c|c|c|c|}
\hline \multirow[t]{2}{*}{ Cancer Site (ICD-O-3) } & \multicolumn{3}{|l|}{ Men } & \multicolumn{3}{|c|}{ Women } \\
\hline & Cases & $\mathrm{HR}$ & $(95 \% C l)^{a}$ & Cases & $\mathrm{HR}$ & $(95 \% \mathrm{Cl})^{\mathrm{a}}$ \\
\hline Any cancer ${ }^{b}$ & 240 & 0.94 & $(0.83-1.07)$ & 160 & 0.90 & $\overline{(0.77-1.05)}$ \\
\hline Prostate (C61.9) & 90 & 1.26 & $(1.03-1.55)$ & - & - & - \\
\hline Breast (C50) & 0 & - & - & 45 & 0.76 & $(0.57-1.02)$ \\
\hline Lung (C34) & 30 & 0.69 & $(0.48-0.98)$ & 20 & 0.61 & $(0.37-0.99)$ \\
\hline Colon (C18, C26.0) & 20 & 0.97 & $(0.63-1.49)$ & 20 & 1.35 & $(0.87-2.09)$ \\
\hline Rectum (C19.9, C20.9) & 10 & 0.93 & $(0.54-1.60)$ & 5 & 0.86 & $(0.36-2.06)$ \\
\hline Non-Hodgkin Lymphoma ${ }^{c}$ & 15 & 0.89 & $(0.54-1.45)$ & 5 & 0.72 & $(0.33-1.61)$ \\
\hline Melanoma (C44) & 15 & 1.44 & $(0.82-2.53)$ & 5 & 1.11 & $(0.53-2.33)$ \\
\hline Thyroid (C73.9) & 10 & 3.01 & $(1.35-6.73)$ & 10 & 1.28 & $(0.57-2.85)$ \\
\hline Oral (COO-C14) & 10 & 1.16 & $(0.62-2.15)$ & 5 & 2.70 & $(1.28-5.68)$ \\
\hline Lip (C00.0-C00.9) & 5 & 2.91 & $(1.08-7.80)$ & $<5$ & - & - \\
\hline Stomach (C16) & 10 & 1.22 & $(0.63-2.35)$ & $<5$ & - & - \\
\hline Bladder (C67) & 10 & 0.72 & $(0.40-1.31)$ & $<5$ & - & - \\
\hline Kidney (C64.9) & 5 & 0.57 & $(0.26-1.27)$ & $<5$ & - & - \\
\hline Leukemia $^{c}$ & 5 & 0.82 & $(0.37-1.83)$ & $<5$ & - & - \\
\hline Pancreas (C25) & $<5$ & - & - & 5 & 1.14 & $(0.51-2.54)$ \\
\hline Cervix (C53) & - & - & - & 5 & 1.55 & $(0.70-3.46)$ \\
\hline Larynx (C32) & 5 & 1.46 & $(0.61-3.52)$ & 0 & - & - \\
\hline Multiple Myeloma ${ }^{c}$ & $<5$ & - & - & $<5$ & - & - \\
\hline Esophagus (C15) & $<5$ & - & - & $<5$ & - & - \\
\hline Liver (C22.0, C22.1) & $<5$ & - & - & $<5$ & - & - \\
\hline Brain (C70-C72) & $<5$ & - & - & $<5$ & - & - \\
\hline Nasal (C30) & $<5$ & - & - & 0 & - & - \\
\hline Bone (C40, C41) & $<5$ & - & - & 0 & - & - \\
\hline Hodgkin Lymphoma $^{c}$ & $<5$ & - & - & 0 & - & - \\
\hline Ovary (C56.9) & - & - & - & $<5$ & - & - \\
\hline Testis (C62) & 0 & - & - & - & - & - \\
\hline Mesothelioma ${ }^{c}$ & 0 & - & - & 0 & - & - \\
\hline
\end{tabular}

Note: case counts below 5 have been suppressed and all counts have been randomly rounded to base 5 in accordance with Statistics Canada disclosure rules ${ }^{a}$ Adjusted for age at baseline (age group categories), province of residence at baseline, and education level at baseline

${ }^{b}$ Incident primary cancers excluding non-melanoma skin cancer

'Cancers defined using ICD-O-3 Histology codes: Mesothelioma (9050-9055), Hodgkin lymphoma (9650-9667); non-Hodgkin lymphoma (9590-9596,9670-9719, 9727-9729, 9823, 9827); Multiple myeloma (9731,9732,9734); Leukemia (9733, 9742, 9800-9801, 9805, 9820, 9826, 9831-9837, 9840, 9860-9861, 9863, 9866-9867, 9870-9876,9891,9895-9897,9910, 9920, 9930-9931, 9940, 9945-9946, 9948, 9963-9964, 9823, 9827)

greater for agricultural workers overall. Excess risks of thyroid cancer were observed for all women in agriculture $(\mathrm{HR}=1.26,95 \% \mathrm{CI}: 0.99-1.60)$, and brain cancer incidence appeared elevated among female farmers and managers (HR $=1.45,95 \%$ CI: 0.96-2.21).

In our next sensitivity analysis, we estimated risks associated with agricultural occupation for selected cancers separately among participants aged 25 to 44 years old at enrollment and those aged 45 to 74 years on census day (Additonal file 3: Table S3). As expected, the number of cancers diagnosed in the older age group (7945 cases) was higher than the number of cases observed among younger participants (1570 cases). Increased risks of leukemia $(\mathrm{HR}=1.30,95 \% \mathrm{CI}$ : 1.15-1.47), multiple myeloma $(\mathrm{HR}=1.22,95 \% \mathrm{CI}$ : $1.01-1.46)$, prostate $(\mathrm{HR}=1.26,95 \% \mathrm{CI}: 1.21-1.32)$ and thyroid cancer $(\mathrm{HR}=1.34,95 \% \mathrm{CI}: 1.07-1.72)$ were limited to agricultural workers in the older age stratum.

However, the increased risk of lip cancer identified in the main analyses was observed among agricultural workers aged 25 to 44 years ( $\mathrm{HR}=2.38,95 \% \mathrm{CI}: 1.32-4.28)$, as well as in the older age stratum ( $\mathrm{HR}=2.28,95 \% \mathrm{CI}: 1.79-2.91)$. Melanoma risks were only $8 \%$ greater among younger 
participants, but risks associated with agricultural work were significantly elevated by $25 \%$ among workers aged 45 to 74 years. Excess risk of NHL was observed among agricultural workers aged 45 to 74 years $(\mathrm{HR}=1.19,95 \% \mathrm{CI}$ : 1.08-1.30) and this association was attenuated in the younger age group $(\mathrm{HR}=1.14,95 \% \mathrm{CI}: 0.95-1.37)$.

\section{Discussion}

This analysis of a large, population-based cohort study of Canadian agricultural workers has confirmed many established patterns of cancer risk, and uncovered several novel associations. As expected, overall cancer risk was significantly lower for both men and women employed in agricultural occupations, in comparison with the rest of the working population. The incidence of main tobacco- and alcohol-related cancers, such as lung, liver and larynx was also significantly reduced for both men and women working in agriculture.

However, a cautious interpretation of our findings is warranted since this cohort was designed for the purposes of cancer surveillance, with the primary goal of identifying meaningful patterns in cancer incidence by occupation. Therefore, while this analysis sacrifices some detail and specificity compared to exposure assessments in studies designed to evaluate specific agricultural exposures, linkage projects such as this one provide a unique opportunity to examine cancer patterns by occupation for a large, nationally representative sample of the population. Although our findings do not elucidate specific exposure-response relationships, the associations identified in this cohort provide leads regarding exposures that may be implicated in cancer risk and warrant follow-up in other studies.

Pesticides, several of which have been classified as known (Group 1) or probable (Group 2A) human carcinogens by the International Agency for Research on Cancer and several regulatory agencies in the United States [23-25], are among the most prevalent and studied agricultural exposures [26]. Exposure to pesticides is often considered a major factor underlying increased risks of NHL, multiple myeloma, and leukemia observed in agricultural populations [3, 11]. A meta-analysis of 13 case-control studies observed a significantly increased risk of NHL associated with occupational pesticide exposure, and suggestive associations were reported for other hematopoietic cancers [27]. This pattern of results is consistent with the findings of population-based casecontrols studies of pesticide exposure in Canada [28-30] and the United States [31-33], as well as results from the AHS cohort [10].

Our findings of increased leukemia risk among female farmers and managers, and specifically crop farmers, are also supported by recent studies of farming and pesticide exposure. The Iowa Women's Health Study, a large cohort created by linkage to the Iowa Cancer Registry, reported increased risks of acute myeloid leukemia (AML) among women living on farms [34]. Furthermore, a large multi-site prospective cohort of postmenopausal women in the United States also reported increased risks of chronic lymphocytic leukemia/small lymphocytic lymphoma and AML associated with insecticide exposure [35]. Therefore, while the excess risks of hematological cancers observed in our study cannot be directly attributed to a specific exposure, the existing epidemiologic literature is suggestive of pesticides as a contributing factor.

The increased risk of pancreatic cancer observed among women employed in agriculture is a noteworthy finding of this study. A Spanish case-control study observed similarly increased risks of pancreatic cancer among female, but not male, agricultural workers [36]. However, a later analysis of a similar population-based case-control study in Spain observed a non-significant suggestive increase in risk male agricultural workers only [37]. A meta-analysis of occupational risk factors for pancreatic cancer identified several positive but weak associations, with the strongest evidence observed for nickel compounds based on results in four populations [38].

Pesticide exposure has been linked to prostate cancer $[39,40]$, however, the overall evidence for an increased risk of prostate cancer in farmers remains weak [41] and is unlikely to account for the modestly increased risks observed in our study. The observed associations may reflect a multitude of factors, including genetic predisposition and screening behavior, and should be interpreted with caution since the etiology of prostate cancer is poorly understood. Currently, the only established risk factors for prostate cancer are age, ethnicity, and a positive family history of prostate cancer [42]. Despite extensive research into lifestyle factors, the epidemiological evidence remains mixed. Early observations of increased prostate cancer risk associated with reduced sunlight exposure prompted investigations into the protective effects of vitamin D [43]. Although it was initially promoted for prostate cancer prevention [44], the accumulation of convicting findings, and recent reviews and meta-analyses of vitamin D do not support a convincing causal relationship with prostate cancer incidence [45-47].

The inverse risk estimates observed for kidney, colon, rectal and bladder cancers are suggestive of a favourable risk factor profile in agricultural workers with respect to physical activity and body weight, which are recognized modifiable risk factors for these cancers $[48,49]$. The decreased risks of bladder cancer observed consistently among male agricultural workers may also be related to the low prevalence of cigarette smoking in this population [50]. However, the impact of other environmental 
and occupational bladder cancer risk factors, such as contamination of drinking water with arsenic, exposure to polycyclic aromatic hydrocarbons (PAHs), aromatic amines, and diesel and gasoline emissions cannot be discounted [51-53].

In addition to a low prevalence of tobacco smoking, endotoxin exposure may also contribute to the lower risk of lung cancer observed in agricultural workers. Endotoxin, or lipopolysaccharide (LPS), is a component of the outer membrane of Gram-negative bacteria, and is released during cell replication. These molecules are ubiquitous and inhalation of endotoxins present in dust is the main route of exposure. High levels of endotoxin exposure have been documented in agricultural settings, especially for activities involving animal breeding and handling [54]. Although the epidemiologic studies do not provide strong causal evidence, some mechanistic studies suggest that endotoxins can inhibit tumor initiation and growth, and LPS may stimulate the production of endogenous antineoplastic mediators [55-57]. A systematic review of cohort and case control studies of lung cancer in cotton textile production and agriculture found significant inverse associations for endotoxin exposure among livestock farmers [58], suggesting that it may partly contribute to the lower risk of lung cancer observed for some agricultural occupations.

Our findings of statistically significant increases in the risk of lip cancer and melanoma among men implicate exposure to sunlight and ultraviolet (UV) radiation as a putative risk factor. Studies have identified UV exposure as a major determinant of both melanoma and nonmelanoma skin cancers, including cancers of the facial skin [59]. Due to the small number of lip cancers in women this association could not be estimated, although excesses in melanoma risk, especially among female crop farmers, are compatible with this hypothesis. Overall, it appears that occupations associated high socioeconomic status, as well as jobs that involve outdoor work and potential for exposure to industrial chemicals tend to show increased risks of skin and lip cancer, compared with the general population $[7,60]$. However, increased melanoma risk among agricultural workers may also be related to pesticide exposure, with studies reporting associations both with occupational [61] and residential use [62].

Cancers of the oral cavity are a diverse group of tumours arising from the epithelium lining the oral cavity and pharynx, with distinct risk factor profiles. The increased risks observed for lip cancer specifically, rather than all oral cavity cancers, is likely to reflect the interplay between risk factors shared common to all oral carcinomas, such as alcohol consumption and tobacco smoking, and more specific causal factors, such as human papillomavirus infection, a susceptibility factor for oropharyngeal carcinoma $[63,64]$.
Our analysis of more refined agricultural subgroups also revealed a 3-fold increased risk of thyroid cancer among male crop farmers. There are few known thyroid cancer risk factors with the exception of female sex and ionizing radiation. Although thyroid hormone excretion and metabolism can be disrupted by a number of chemicals found in the workplace, such as organochlorine pesticides, polychlorinated biphenyls, and polybrominated diphenyl ethers, the link with thyroid cancer remains tenuous. An analysis of predominantly male atrazine users in the AHS found an elevated risk of thyroid cancer for the highest compared to the lowest category of intensity-weighted use, but the trend was not monotonic and not statistically significant [65].

Several limitations of this analysis should be acknowledged. Given the retrospective nature of this cohort and its creation using linkage between existing health and administrative data sources, we did not have information on duration of employment in agriculture prior to the 1991 Census. We were also unable to track changes in occupation over the course of the follow-up period. This relatively crude approach is likely to reduce the specificity and sensitivity of our exposure assessment, resulting in exposure misclassification. However, since these limitations apply to all occupational groups within the cohort, and errors in exposure ascertainment are independent of cancer status, comparisons between agricultural workers and other groups may lead to imprecise risk estimates, but are unlikely to produce spurious associations [66]. Furthermore, classifying individuals with a short duration of employment in agriculture as exposed, would be expected to bias associations towards the null.

A second limitation concerns the possibility that some participants were diagnosed with cancer prior to cohort inception in 1991. Failure to account for a past history of cancer would only bias our analyses if agricultural workers as a group were enriched for cancer survivors, compared to all other occupations. However, since this is unlikely, a more relevant concern is that some prevalent cancer cases in the cohort were classified as outcome-free. This would be expected to bias the observed associations towards the null if the misclassification of disease status is non-differential with respect to occupation. While we acknowledge that a pre-1991 cancer diagnosis may affect the participant's subsequent employment trajectory and occupation reported on census day, these effects are unlikely to have a differential impact on workers in agriculture compared to other industries [67].

Furthermore, our sensitivity analyses demonstrate that most of the observed associations persisted after the exclusion of individuals diagnosed with cancer within 10 years of cohort inception. The excess risks of lip cancer, melanoma and NHL in men were also observed in 
the sub-cohort, along with the inverse associations for lung, liver, bladder and larynx cancers. Similarly, the array of increased and decreased risks observed for female agricultural workers in the main analysis were also observed in the sensitivity analyses. Therefore, the stable pattern of associations emerging in both the full CanCHEC cohort and the cancer-free sub-cohort suggests that the inclusion of pre-1991 cancer cases is unlikely to bias the observed cancer risks or create spurious associations.

Confounding by lifestyle-related cancer risk factors is often a concern in occupational studies, as well as in studies relying on linkages between cancer registries and existing databases. Although smoking information was not available in CanCHEC, previous studies, in addition to analyses using the same linked 1991 Census data show that major determinants of smoking status include age, sex, level of education and occupation [21, 22]. Therefore, although confounding by smoking cannot be excluded, adjustment for key determinants of smoking status in our analysis helps mitigate the impact of this residual confounding. Furthermore, the magnitude of confounding by tobacco smoking or high levels of alcohol consumption may be further minimized in our study due to the low prevalence of both of these risk factors in agricultural populations, contemporaneous to CanCHEC inception $[68,69]$.

Other limitations of this study include limited power for investigating less common cancers, especially within more refined agricultural subgroups. In addition, given the large number of cancer sites and occupational subgroups that were tested, some of the observed associations may be chance findings due to multiple comparisons.

Our study has a number of important strengths. CanCHEC is the largest population-based cohort in Canada, and one of the largest studies of its kind worldwide. Although it is smaller than the previously mentioned NOCCA cohort of 15 million [7], the 2.1 million participants in CanCHEC, including 70,570 agricultural workers are broadly representative of the Canadian population due to the high response rate for the 1991 Census [15]. Although the exposure assessment is less detailed than in studies such as the AHS, CanCHEC provides a powerful resource for examining cancer patterns in specific population groups and represents a valuable addition to the cancer literature by demonstrating that disparities in cancer risk by occupation status continue to persist and should be investigated using more targeted studies. The updated linkage to the Canadian Cancer Registry allows for a long follow-up period, which is important for cancer outcomes that have long latency periods. Together, the large sample size and length of follow-up allow for a large number of events, which results in improved statistical power for the investigation of detailed occupational subgroups and specific cancer sites. Importantly, relatively few studies focus on women employed in agriculture, and even fewer have systematically reported estimates of cancer risk across multiple sites and occupational subgroups.

In the beginning twentieth century, agriculture was the single most common occupation, employing over 1 million Canadians and accounting for one-third of all jobs. Over the course of our follow-up period, between 1991 and 2011, the total number of Canadian farms fell by over 74,000 and now represents less than $1 \%$ of the labour force. However, if agricultural populations experience higher incidence for certain types of cancer, understanding the magnitude of these increased risks represents an important step towards developing preventive efforts targeting these populations.

Despite the changing profile of Canadian agricultural workers, studies of this population continue to be relevant by providing insight into cancer risks associated with common exposures that are often found outside of agricultural settings. For instance, the large magnitude of UV-related risks, especially for lip cancer, underscores the substantial impact that may be achieved by preventive efforts in this area. Therefore, studies of agricultural workers can inform preventive interventions aimed at reducing the cancer burden in the general population.

\section{Conclusions}

In summary, the results of the present analysis point to excess risks for certain cancers among agricultural workers. A wide range of exposures is possible in agriculture, including pesticides, solvents, engine exhaust emission, UV light, dust, as well as zoonotic viruses and bacteria. Exposures can vary considerably between occupations, and even between farms, therefore future research must focus on specific exposures to identify and clarify which risk factors may contribute to the observed pattern of cancer incidence.

\section{Additional files}

\section{Additional file 1: Table S1. Hazard ratios (HR) and 95\% confidence intervals $(\mathrm{Cl})$ for selected cancers among male agricultural workers in a sub- cohort of CanCHEC that excludes all individuals with a cancer diagnosis within 10 years of cohort inception (1981-1991). (PDF 84 kb) \\ Additional file 2: Table S2. Hazard ratios (HR) and 95\% confidence intervals $(\mathrm{Cl})$ for selected cancers among female agricultural workers in a sub-cohort of CanCHEC that excludes all individuals with a cancer diagnosis within 10 years of cohort inception (1981-1991). (PDF 84 kb)}

Additional file 3: Table S3. Hazard ratios (HR) and 95\% confidence intervals (Cl) for selected cancers with more than 100 cases among agricultural workers in CanCHEC (1991-2010), stratified by age at enrollment in 1991 (1981-1991). (PDF 53 kb)

\section{Abbreviations}

AHS: Agricultural health study; AML: Acute myeloid leukemia; CanCHEC: Canadian Census Health and Environment Cohort; CCR: Canadian cancer registry; CMDB: Canadian mortality database; HR: Hazard ratio; 
Cl: Confidence intervals; IARC: International Agency for Research on Cancer; ICD-O: International classification of diseases for oncology; LPS: Lipopolysaccharide; NHL: Non-Hodgkin lymphoma; SOC: Standard occupational classification; UV: Ultraviolet radiation

\section{Acknowledgements}

This research was supported by the Canadian Research Data Centre Network from the Social Science and Humanities research Council, the Canadian Institute for Health Research, the Canadian Foundation for Innovation, and Statistics Canada.

\section{Funding}

Linkage of the 1991 Canadian Census to the Canadian Cancer Registry was funded by the Canadian Institute for Health Information, Health Canada and Statistics Canada. This study was supported by the Workers Safety and Insurance Board of Ontario (Grant \#11024).

\section{Availability of data and materials}

Data from the 1991 Canadian Census Cohort are available for researchers approved by Statistics Canada, within the Canadian Research Data Centre Network (http://www.rdc-cdr.ca/). Access is also available to external researchers through the Centre for Data Development and Economic Research (CDER) at Statistics Canada (http://www.statcan.gc.ca/cder-cdre/ index-eng.htm) Researchers and organizations can also seek to work collaboratively with analysts at Statistics Canada. More information can be obtained by contacting Statistics Canada at statcan.had-das-dashad.statcan@canada.ca

\section{Authors' contributions}

Conception and design of the CanCHEC study, including data linkage and acquisition were lead and carried out by MT, PAP, and PAD. Conception and design of this analysis were carried out by MAH, LK and PAD. Exposure assessment was lead by LK, MAH and PAD. Data analysis was conducted by LK and JSM. Data interpretation and drafting of the manuscript was lead by LK. All authors participated in revising the manuscript for important intellectual content. All authors read and approved the final manuscript.

\section{Competing interests}

The authors declare no competing interests. No payment or services received from a third party. No relevant financial activities outside the submitted work. No patents (planned, pending, or issued) broadly relevant to this work. No other relationships or conditions or circumstances that present a potential conflict of interest.

\section{Consent for publication}

Not Applicable.

\section{Ethics approval and consent to participate}

This study was approved by the University of Toronto's Health Sciences Research Ethics Board. This linkage project was approved by the Statistics Canada Executive Management Board after consultation with Statistics Canada Confidentiality and Legislation Committee, Data Access and Contro Services Division, and the Federal Privacy Commissioner. Although the research and analysis are based on data from Statistics Canada, the opinions expressed do not represent the views of Statistics Canada or the Canadian Research Data Centre Network

\section{Publisher's Note}

Springer Nature remains neutral with regard to jurisdictional claims in published maps and institutional affiliations.

\section{Author details}

'Dalla Lana School of Public Health, University of Toronto, 155 College Street, 6th Floor, Toronto, ON M5T 3M7, Canada. ${ }^{2}$ Occupational Cancer Research Centre, Cancer Care Ontario, 525 University Avenue, 3rd Floor, Toronto, ON M5G 2L3, Canada. ${ }^{3}$ Prevention and Cancer Control, Cancer Care Ontario, 620 University Ave, Toronto, ON M5G 2L7, Canada. ${ }^{4}$ School of Occupational and Public Health, Ryerson University, 350 Victoria Street, POD 249, Toronto, ON M5B 2K3, Canada. ${ }^{5}$ Health Analysis Division, Statistics Canada, 150 Tunney's Pasture Driveway, Ottawa, ON K1A 0T6, Canada. 'Departments of Sociology and Economics, University of New Brunswick, Tilley Hall 015, 9 Macaulay Lane, Fredericton, NB E3B 5A3, Canada.
Received: 26 June 2016 Accepted: 11 May 2017 Published online: 19 May 2017

\section{References}

1. Blair A, Malker H, Cantor KP, Burmeister L, Wiklund K. Cancer among farmers. A review. Scand J Work Environ Health. 1985;11(6):397-407.

2. Blair A, Zahm SH, Pearce NE, Heineman EF, Fraumeni JF Jr. Clues to cance etiology from studies of farmers. Scand J Work Environ Health. 1992;18(4): 209-15

3. Acquavella J, Olsen G, Cole P, Ireland B, Kaneene J, Schuman S, Holden L. Cancer among farmers: a meta-analysis. Ann Epidemiol. 1998;8(1):64-74.

4. Pearce N, Reif JS. Epidemiologic studies of cancer in agricultural workers. Am J Ind Med. 1990;18(2):133-48.

5. Laakkonen A, Pukkala E. Cancer incidence among Finnish farmers, 1995 2005. Scand J Work Environ Health. 2008;34(1):73-9.

6. Blair A, Walrath J, Rogot E. Mortality patterns among U.S. veterans by occupation. I. Cancer. J Natl Cancer Inst. 1985;75(6):1039-47.

7. Pukkala E, Martinsen Jl, Lynge E, Gunnarsdottir HK, Sparen P, Tryggvadottir L, Weiderpass E, Kjaerheim K. Occupation and cancer - follow-up of 15 million people in five Nordic countries. Acta Oncol. 2009;48(5):646-790.

8. Neugut Al, Terry MB, Hocking G, Mosca L, Garbowski GC, Forde KA, Treat MR, Waye J. Leisure and occupational physical activity and risk of colorectal adenomatous polyps. Int J Cancer. 1996;68(6):744-8.

9. Pfeiffer S, Graham TE, Webb RD, Wilson BA, Rivington-Moss EG, FisherIngram LM. Aspects of physical fitness and health in Ontario dairy farmers Can J Public Health. 1984;75(3):204-11.

10. Koutros S, Alavanja MC, Lubin JH, Sandler DP, Hoppin JA, Lynch CF, Knott C, Blair A, Freeman LE. An update of cancer incidence in the agricultural health study. J Occup Environ Med. 2010;52(11):1098-105.

11. Blair A, Freeman LB. Epidemiologic studies in agricultural populations: observations and future directions. J Agromedicine. 2009;14(2):125-31.

12. Blair A. Occupation and cancer in the Nordic countries. Acta Oncol. 2009; 48(5):644-5.

13. Leon ME, Beane Freeman LE, Douwes J, Hoppin JA, Kromhout H, Lebailly $P$, Nordby KC, Schenker M, Schuz J, Waring SC, et al. AGRICOH: a consortium of agricultural cohorts. Int J Environ Res Public Health. 2011;8(5):1341-57.

14. Wilkins R, Tjepkema M, Mustard C, Choiniere R. The Canadian census mortality follow-up study, 1991 through 2001. Health Rep. 2008;19(3):25-43.

15. Peters PA, Tjepkema M, Wilkins R, Fines P, Crouse DL, Chan PC, Burnett RT Data resource profile: 1991 Canadian census cohort. Int J Epidemiol. 2013; 42(5):1319-26.

16. Carpenter M, Fair ME, Poliquin C, Lalonde P. Canadian cancer Data Base, 1969 to 1991 history and development. In: Vol. report no. 16: health Statistics Division, Statistics Canada; 2008.

17. International Agency for Research on Cancer. International rules for multiple primary cancers. Lyon: IARC, World Health Organization; 2004.

18. Soskolne $\mathrm{CL}$, Sieswerda LE. Cancer risk associated with pulp and paper mills: a review of occupational and community epidemiology. Chronic Dis Can. 2010;29(Suppl 2):86-100.

19. Rostgaard K. Methods for stratification of person-time and events - a prerequisite for Poisson regression and SIR estimation. Epidemiol Perspect Innov. 2008;5:7.

20. Dickman PW, Sloggett A, Hills M, Hakulinen T. Regression models for relative survival. Stat Med. 2004;23(1):51-64.

21. Winkleby MA, Jatulis DE, Frank E, Fortmann SP. Socioeconomic status and health: how education, income, and occupation contribute to risk factors for cardiovascular disease. Am J Public Health. 1992;82(6):816-20.

22. Sanmartin C, Fines $P$, Khan S, Peters $P$, Tjepkema M, Bernier J, Burnett R. Modelling risk factor information for linked census data: the case of smoking. Health Rep. 2013;24(6):9-15.

23. International Agency for Research on Cancer. Occupational exposures in insecticide application, and some pesticides. In: IARC monographs on the evaluation of carcinogenic risks to humans, vol. 53. Lyon: World Health Organization; 1991.

24. International Agency for Research on Cancer. Some organophosphate insecticides and herbicides: Diazinon, Glyphosate, Malathion, parathion, and Tetrachlorvinphos. In: IARC monographs on the evaluation of carcinogenic risks to humans, vol. 112. Lyon: World Health Organization; 2015.

25. National Toxicology Program. Report on carcinogens, thirteenth edition. Research Triangle Park: Department of Health and Human Services; 2014. 
26. Brouwer M, Schinasi L, Beane Freeman LE, Baldi I, Lebailly P, Ferro G, Nordby KC, Schuz J, Leon ME, Kromhout H. Assessment of occupational exposure to pesticides in a pooled analysis of agricultural cohorts within the AGRICOH consortium. Occup Environ Med. 2016;73(6):359-67.

27. Merhi M, Raynal H, Cahuzac E, Vinson F, Cravedi JP, Gamet-Payrastre L. Occupational exposure to pesticides and risk of hematopoietic cancers: metaanalysis of case-control studies. Cancer Causes Control. 2007;18(10):1209-26.

28. McDuffie HH, Pahwa P, McLaughlin JR, Spinelli JJ, Fincham S, Dosman JA, Robson D, Skinnider LF, Choi NW. Non-Hodgkin's lymphoma and specific pesticide exposures in men: cross-Canada study of pesticides and health. Cancer Epidemiol Biomark Prev. 2001;10(11):1155-63.

29. Kachuri L, Demers PA, Blair A, Spinelli JJ, Pahwa M, McLaughlin JR, Pahwa P, Dosman JA, Harris SA. Multiple pesticide exposures and the risk of multiple myeloma in Canadian men. Int J Cancer. 2013;133(8):1846-58.

30. Hohenadel K, Harris SA, McLaughlin JR, Spinelli JJ, Pahwa P, Dosman JA, Demers PA, Blair A. Exposure to multiple pesticides and risk of nonHodgkin lymphoma in men from six Canadian provinces. Int J Environ Res Public Health. 2011;8(6):2320-30.

31. Brown LM, Blair A, Gibson R, Everett GD, Cantor KP, Schuman LM, Burmeister LF, Van Lier SF, Dick F. Pesticide exposures and other agricultural risk factors for leukemia among men in lowa and Minnesota. Cancer Res. 1990;50(20):6585-91.

32. Cantor KP, Blair A, Everett G, Gibson R, Burmeister LF, Brown LM, Schuman L, Dick FR. Pesticides and other agricultural risk factors for non-Hodgkin's lymphoma among men in lowa and Minnesota. Cancer Res. 1992;52(9):2447-55.

33. Brown LM, Burmeister LF, Everett GD, Blair A. Pesticide exposures and multiple myeloma in lowa men. Cancer Causes Control. 1993:4(2):153-6.

34. Jones RR, Yu CL, Nuckols JR, Cerhan JR, Airola M, Ross JA, Robien K, Ward $\mathrm{MH}$. Farm residence and lymphohematopoietic cancers in the lowa Women's health study. Environ Res. 2014;133:353-61.

35. Schinasi LH, De Roos AJ, Ray RM, Edlefsen KL, Parks CG, Howard BV, Meliker JR, Bonner MR, Wallace RB, LaCroix AZ. Insecticide exposure and farm history in relation to risk of lymphomas and leukemias in the Women's Health Initiative observational study cohort. Ann Epidemiol. 2015;25(11):803-10.

36. Alquacil J, Porta M, Benavides FG, Malats N, Kogevinas M, Fernandez E, Carrato A, Rifa J, Guarner L. Occupation and pancreatic cancer in Spain: a case-control study based on job titles. PANKRAS II study group. Int J Epidemiol. 2000;29(6):1004-13.

37. Santibanez M, Vioque J, Alguacil J, de la Hera MG, Moreno-Osset E, Carrato A, Porta M, Kauppinen T. Occupational exposures and risk of pancreatic cancer. Eur J Epidemiol. 2010;25(10):721-30.

38. Ojajarvi IA, Partanen TJ, Ahlbom A, Boffetta P, Hakulinen T, Jourenkova N, Kauppinen TP, Kogevinas M, Porta M, Vainio HU, et al. Occupational exposures and pancreatic cancer: a meta-analysis. Occup Environ Med. 2000;57(5):316-24.

39. Koutros S, Beane Freeman LE, Lubin JH, Heltshe SL, Andreotti G, Barry KH, DellaValle CT, Hoppin JA, Sandler DP, Lynch CF, et al. Risk of total and aggressive prostate cancer and pesticide use in the agricultural health study. Am J Epidemiol. 2013;177(1):59-74.

40. Van Maele-Fabry G, Libotte V, Willems J, Lison D. Review and meta-analysis of risk estimates for prostate cancer in pesticide manufacturing workers. Cancer Causes Control. 2006:17(4):353-73.

41. Depczynski J, Lower T. A review of prostate cancer incidence and mortality studies of farmers and non-farmers, 2002-2013. Cancer Epidemiol. 2014;38(6):654-62

42. Leitzmann MF, Rohrmann S. Risk factors for the onset of prostatic cancer: age, location, and behavioral correlates. Clin Epidemiol. 2012;4:1-11.

43. Hanchette CL, Schwartz GG. Geographic patterns of prostate cancer mortality. Evidence for a protective effect of ultraviolet radiation. Cancer. 1992:70(12):2861-9.

44. Li H, Stampfer MJ, Hollis JB, Mucci LA, Gaziano JM, Hunter D, Giovannucci EL, Ma J. A prospective study of plasma vitamin $D$ metabolites, vitamin D receptor polymorphisms, and prostate cancer. PLoS Med. 2007:4(3):e103

45. Gandini S, Boniol M, Haukka J, Byrnes G, Cox B, Sneyd MJ, Mullie P, Autier P. Meta-analysis of observational studies of serum 25 -hydroxyvitamin $D$ levels and colorectal, breast and prostate cancer and colorectal adenoma. Int Cancer. 2011;128(6):1414-24.

46. Gilbert R, Martin RM, Beynon R, Harris R, Savovic J, Zuccolo L, Bekkering GE, Fraser WD, Sterne JA, Metcalfe C. Associations of circulating and dietary vitamin $D$ with prostate cancer risk: a systematic review and dose-response meta-analysis. Cancer Causes Control. 2011;22(3):319-40.
47. Feldman D, Krishnan AV, Swami S, Giovannucci E, Feldman BJ. The role of vitamin D in reducing cancer risk and progression. Nat Rev Cancer. 2014;14(5):342-57.

48. Moore SC, Lee IM, Weiderpass E, Campbell PT, Sampson JN, Kitahara CM, Keadle SK, Arem H, Berrington de Gonzalez A, Hartge P, et al. Association of Leisure-Time Physical Activity with Risk of 26 types of cancer in 1.44 million adults. JAMA Intern Med. 2016;176(6):816-25.

49. Brenner DR. Cancer incidence due to excess body weight and leisure-time physical inactivity in Canada: implications for prevention. Prev Med. 2014;66:131-9.

50. Zeegers MP, Kellen E, Buntinx F, van den Brandt PA. The association between smoking, beverage consumption, diet and bladder cancer: a systematic literature review. World J Urol. 2004;21(6):392-401.

51. Zeegers MP, Swaen GM, Kant I, Goldbohm RA, van den Brandt PA. Occupational risk factors for male bladder cancer: results from a population based case cohort study in the Netherlands. Occup Environ Med. 2001:58(9):590-6.

52. Latifovic L, Villeneuve PJ, Parent ME, Johnson KC, Kachuri L, Canadian cancer registries epidemiology G, Harris SA. Bladder cancer and occupational exposure to diesel and gasoline engine emissions among Canadian men. Cancer Med. 2015;4(12):1948-62.

53. Letasiova S, Medve'ova A, Sovcikova A, Dusinska M, Volkovova K, Mosoiu C, Bartonova A. Bladder cancer, a review of the environmental risk factors. Environ Health. 2012:11(Suppl 1):S11.

54. Liebers V, Bruning T, Raulf-Heimsoth M. Occupational endotoxin-exposure and possible health effects on humans. Am J Ind Med. 2006;49(6):474-91.

55. Reisser D, Pance A, Jeannin JF. Mechanisms of the antitumoral effect of lipid a. BioEssays. 2002;24(3):284-9.

56. Pance A, Reisser D, Jeannin JF. Antitumoral effects of lipid a: preclinical and clinical studies. J Investig Med. 2002;50(3):173-8.

57. Chicoine MR, Won EK, Zahner MC. Intratumoral injection of lipopolysaccharide causes regression of subcutaneously implanted mouse glioblastoma multiforme. Neurosurgery. 2001;48(3):607-14. discussion 614-605

58. Lenters V, Basinas I, Beane-Freeman L, Boffetta P, Checkoway H, Coggon $D$, Portengen L, Sim M, Wouters IM, Heederik D, et al. Endotoxin exposure and lung cancer risk: a systematic review and meta-analysis of the published literature on agriculture and cotton textile workers. Cancer Causes Control. 2010;21(4):523-55.

59. Rigel DS. Cutaneous ultraviolet exposure and its relationship to the development of skin cancer. J Am Acad Dermatol. 2008;58(5 Suppl 2): S129-32.

60. Alfonso JH, Martinsen Jl, Pukkala E, Weiderpass E, Tryggvadottir L, Nordby KC, Kjaerheim K. Occupation and relative risk of cutaneous squamous cell carcinoma (CSCC): a 45-year follow-up study in 4 Nordic countries. J Am Acad Dermatol. 2016:75(3):548-55.

61. Dennis LK, Lynch CF, Sandler DP, Alavanja MC. Pesticide use and cutaneous melanoma in pesticide applicators in the agricultural heath study. Environ Health Perspect. 2010;118(6):812-7

62. Fortes C, Mastroeni S, Melchi F, Pilla MA, Alotto M, Antonelli G, Camaione D, Bolli S, Luchetti E, Pasquini P. The association between residential pesticide use and cutaneous melanoma. Eur J Cancer. 2007:43(6):1066-75.

63. Curado MP, Hashibe M. Recent changes in the epidemiology of head and neck cancer. Curr Opin Oncol. 2009;21(3):194-200.

64. Gillison ML, Chaturvedi AK, Anderson WF, Fakhry C. Epidemiology of human Papillomavirus-positive head and neck Squamous cell carcinoma. J Clin Oncol. 2015:33(29):3235-42.

65. Freeman LE, Rusiecki JA, Hoppin JA, Lubin JH, Koutros S, Andreotti G, Zahm SH, Hines CJ, Coble JB, Barone-Adesi F, et al. Atrazine and cancer incidence among pesticide applicators in the agricultural health study (1994-2007). Environ Health Perspect. 2011:119(9):1253-9.

66. Pearce N, Checkoway H, Kriebel D. Bias in occupational epidemiology studies. Occup Environ Med. 2007:64(8):562-8.

67. Jeon $\mathrm{SH}$. The long term effects of cancer on employment and earnings. Health Econ. 2017:26(5):671-84

68. Nelson DE, Emont SL, Brackbill RM, Cameron LL, Peddicord J, Fiore MC. Cigarette smoking prevalence by occupation in the United States. A comparison between 1978 to 1980 and 1987 to. J Occup Med. 1990;36(5): 516-25

69. Mandell W, Eaton WW, Anthony JC, Garrison R. Alcoholism and occupations: a review and analysis of 104 occupations. Alcohol Clin Exp Res. 1992;16(4): 734-46. 